\title{
A MODEL FOR A SPREADING AND MELTING DROPLET ON A HEATED SUBSTRATE*
}

\author{
D. M. ANDERSON ${ }^{\dagger \ddagger}$, M. G. FOREST ${ }^{\dagger}$, AND R. SUPERFINE $§$
}

\begin{abstract}
We develop a model to describe the dynamics of a spreading and melting droplet on a heated substrate. The model, developed in the capillary-dominated limit, is geometrical in nature and couples the contact line, trijunction, and phase-change dynamics. The competition between spreading and melting is characterized by a single parameter $\mathcal{K}_{T}$ that represents the ratio of the characteristic contact line velocity to the characteristic melting (or phase-change) velocity. A key component of the model is an equation of motion for the solid. This equation of motion, which accounts for global effects through a balance of forces over the entire solid-liquid interface, including capillary effects at the trijunction, acts in a natural way as the trijunction condition. This is in contrast to models of trijunction dynamics during solidification, where it is common to specify a trijunction condition based on local physics alone. The trijunction dynamics, as well as the contact angle, contact line position, and other dynamic quantities for the spreading and melting droplet, are predicted by the model and are compared to an isothermally spreading liquid droplet whose dynamics are controlled exclusively by the contact line. We find that in general the differences between the dynamics of a spreading and melting droplet and that of an isothermally spreading droplet increase as $\mathcal{K}_{T}$ increases. We observe that the presence of the solid phase in the spreading and melting configuration tends to inhibit spreading relative to an isothermally spreading droplet of the same initial geometry. Finally, we find that increasing the effect of spreading promotes melting.
\end{abstract}

Key words. contact line, trijunction, droplet spreading, phase transformation

AMS subject classifications. 76D45, 76D27, 80D22, 74N20

PII. S0036139900367188

1. Introduction. The description of a spreading and melting droplet involves both a moving contact line and a trijunction. At the outset, then, we shall distinguish between these two locations and establish the following working definitions. In this work, we refer to a contact line as the intersection of a liquid phase, a vapor phase, and a foreign solid substrate. We refer to a trijunction as the intersection of a liquid phase, its own solid phase, and a vapor phase.

Moving contact lines are ubiquitous in fluid flows that involve interfaces. It is well known that in the continuum description of a contact line moving along a solid substrate with the no-slip condition enforced on the solid substrate, a nonintegrable stress singularity occurs at the contact line (Dussan V. and Davis [1]). Not surprisingly, the problem of a moving contact line has been the subject of mathematical modeling covering macroscopic to molecular scales with descriptions based on continuum mechanics (e.g., Dussan V. [2], Davis [3], de Gennes [4], Ehrhard and Davis [5], Haley and Miksis [6], Hocking [7], Shikhmurzaev [8]), statistical mechanics (e.g., Merchant and Keller [9]), molecular dynamics (e.g., Thompson and Robbins [10], Thompson, Brinckerhoff, and Robbins [11], Koplik and Banavar [12]), and diffuse-interface theories (Jacqmin [13, 14], Seppecher [15]). Experimental work has also covered the broad

\footnotetext{
* Received by the editors January 3, 2000; accepted for publication (in revised form) September 6, 2000; published electronically January 19, 2001.

http://www.siam.org/journals/siap/61-5/36718.html

${ }^{\dagger}$ Department of Mathematics, University of North Carolina, Chapel Hill, NC 27599 (danders1@ gmu.edu, forest@amath.unc.edu).

$\ddagger$ Current address: Department of Mathematical Sciences, George Mason University, Fairfax, VA 22030. The research of this author was supported by the NASA Microgravity Research Division.

$\S$ Department of Physics and Astronomy, University of North Carolina, Chapel Hill, NC 27599 (rsuper@physics.unc.edu).
} 
range of scales from macroscopic (e.g., Ehrhard [16]) to micron-scale (e.g., Marsh, Garoff, and Dussan V. [17], Chen, Ramé, and Garoff [18]) to molecular-scale (e.g., Thiansathaporn [19], Glick [20]).

Contact lines have been examined in isothermal as well as nonisothermal conditions (e.g., Ehrhard and Davis [5], Ehrhard [16], Smith [21]). They may be the location of evaporation (e.g., Moosman and Homsy [22], Wayner [23], Anderson and Davis [24], Hocking [25], Morris and Moreno [26]), as often occurs in the context of heat pipes [27]. Other situations such as the spreading of a reactive material (e.g., Braun et al. [28], Warren, Boettinger, and Roosen [29]), the spreading of a liquid along a porous substrate (Davis and Hocking [30,31]), and the spreading, solidification, and ultimate arrest of a molten contact line (Schiaffino and Sonin [32, 33]) have recently been considered.

Trijunctions, like the contact lines described above, are often the site of phase transformation. In applications such as containerless materials processing techniques, where the melt is partially or completely confined by its own surface tension (e.g., Czochralski growth [34]), a trijunction at which solidification occurs is present. Another materials processing technique, known as float-zone processing, involves trijunctions at both solidifying and melting boundaries (e.g., Surek and Coriell [35]). In this case, a liquid bridge is formed (by thermal control) between two solid ends of a rod in such a way that as the rod is translated along its axis through the heated zone, one side is melted and the other side is solidified. The two solid-liquid interfaces are each bounded by a three-phase line, the trijunction, one of which is the location of solidification and the other of which is the location of melting.

In a problem that is geometrically very similar to the one addressed here, Anderson, Worster, and Davis [36] considered the solidification of a liquid droplet on a cold substrate. They observed that a liquid drop solidified into a solid drop that was cusp-like at the top. Their theoretical predictions showed that the shape was strongly controlled by the conditions imposed at the trijunction. The use of a trijunction condition is typical in problems involving solidification at trijunctions; the most common of these is the "fixed growth angle" condition, where the angle between the tangents of the solid-vapor interface and the liquid-vapor interface at the trijunction is taken to be a constant (e.g., Surek and Chalmers [37], Satunkin, Tatarchenko, and Shaitanov [38], Sanz [39], Brown [34]).

In the present work, we shall describe a spreading and melting droplet for which both a contact line and a trijunction are present. Here, melting occurs at the trijunction as it bounds an evolving solid-liquid interface. Our aim is to develop a mathematical model (using a macroscopic point of view) for the spreading and melting droplet and to identify how the solid phase affects the spreading dynamics.

2. Problem description. We consider the evolution of an initially solid sphere at rest at time zero on a heated substrate. For $t>0$ the droplet melts from the bottom. We assume that gravity is negligible and that the solid portion of the droplet is supported by the liquid layer forming between it and the substrate. (Since gravity is neglected, the problem of spreading on top of a horizontal plate is equivalent to the spreading of a hanging drop on the underside of a horizontal plate.) As the melting proceeds the contact line at the substrate advances. The contact line motion is governed by dynamics which we shall specify locally $[2,5,16]$ but is necessarily coupled through the geometry and the thermal problem to the dynamics of the melting front. In this problem, the solid-liquid interface is defined to be the location of the melting temperature isotherm and the phase transition will be assumed to have associated with 
it zero latent heat. The position of this melting front is controlled not only by the heat transfer through the substrate but also by the dynamics of the underlying liquid layer upon which the solid rides. There is, then, a time scale associated with the contact line dynamics and another associated with the phase transformation dynamics. The ratio of these time scales will play a central role in our model.

The spreading and melting droplet is a transient phenomenon as sufficient heat and time will completely melt droplet and reduce the problem to the spreading of a nonisothermal liquid droplet in the absence of the solid phase (e.g., see Ehrhard and Davis [5]). Hocking and Rivers [40] have addressed the partially melted spreading regime by introducing a shifted time origin for the long-term spreading behavior. Our interest here is specifically on the dynamics when both phases are present.

Our focus on this transient stage has been inspired by experiments performed by Glick [20] on the spreading behavior of polystyrene spheres on glass. In Glick's work, spreading polystyrene spheres (approximately $1 \mathrm{~mm}$ radius) were examined under two different thermal conditions, termed the "oven" and "hot plate" cases. In the oven configuration, a polystyrene sphere was placed at room temperature on a glass substrate that was also at room temperature. The drop and substrate were then placed into an oven whose temperature was fixed above the glass transition temperature of polystyrene. The droplet heated up isothermally and spread. During spreading, the contact angle was measured as a function of time. These measurements were repeated for a range of oven temperatures. A time scale $\mu(T) \mathcal{R}_{0} / \gamma(T)$ based on the ratio of viscosity $\mu(T)$ to surface tension $\gamma(T)$ (both of which are temperature dependent for polystyrene) times the initial droplet radius $\mathcal{R}_{0}$ was identified which allowed the contact angle versus time curves to collapse to a single master curve. The data fit a simple isothermal spreading model based on a spherical cap approximation and a contact line speed versus contact angle relationship (see next section). In the hot plate configuration, a polystyrene sphere at room temperature was placed on a glass substrate whose temperature was already fixed above the glass transition temperature. Here, the droplet spread along the substrate before becoming completely liquid. (To be precise, above the glass transition temperature polystyrene is a relatively mobile viscous fluid, while below the glass transition temperature it is a relatively immobile extremely viscous fluid.) In this configuration, spreading again was observed for a range of substrate temperatures. In this case, the data collapsed to a single curve only when a temperature-dependent length scale $R_{\text {eff }}(T)$ was introduced into the time scale $\mu(T) R_{e f f}(T) / \gamma(T)$. The exact origin and interpretation of $R_{e f f}(T)$ were unclear; however, it was found that $R_{e f f}(T)$ increased with increasing substrate temperature and was in the range of 0.2 to 0.8 times the initial radius of the sphere.

While our modeling has been inspired by the work of Glick in examining these two spreading scenarios, we do not attempt to incorporate non-Newtonian and polymeric aspects into our model. However, we shall adopt the notions of the hot plate and oven configurations in order to assess in a mathematical framework the effect of the presence of the solid phase on contact line spreading. Specifically, we are interested in comparing the evolution from initial conditions of the spreading and melting droplet (hot plate case) to the evolution from initial conditions of an isothermal liquid droplet (oven case) with otherwise the same initial geometry.

Our model is developed in the capillary (or surface tension) dominated limit similar in spirit to those of Ehrhard and Davis [5] and Anderson, Worster, and Davis [36], where the droplet shape dynamics may be decoupled from the bulk fluid hydrodynamic equations of motion. Unlike Ehrhard and Davis, we shall not invoke the 
lubrication approximation but shall rely on sufficiently large surface tension to fix the liquid-vapor interface shape. The resulting model highlights key features of the spreading and melting droplet configuration while avoiding the complexities of solving the bulk hydrodynamic equations of motion for this complicated free-boundary problem.

We begin by outlining in section 3 a model for an isothermal spreading liquid droplet for small capillary number (dominant surface tension). The motivation for doing so is threefold. First, we wish to use the isothermal spreading model to help motivate and develop an analogous spreading and melting model. Second, we shall use the isothermal spreading model to continue the evolution of the spreading and melting droplet once the droplet has completely melted. Third, once a spreading and melting model is solved, we shall use the simple isothermal spreading predictions as a basis for comparison between the two configurations. In section 4 we describe the spreading and melting model, which includes the shape problem and the thermal problem. We then describe the solution method and the model predictions. Following this there is a discussion section and conclusions.

3. Isothermal liquid spreading: Oven configuration. Consider an axisymmetric isothermal liquid droplet for which gravity is zero and the droplet shape is dominated by surface tension (or small capillary number $C=\mu \kappa / \gamma$, where $\mu$ is the fluid viscosity, $\gamma$ is the coefficient of surface tension of the liquid-vapor interface, and $\kappa$ is a characteristic contact line speed defined in (3.3)), as shown in Figure 4.1a. In this situation, arguably one of the simplest to consider, the droplet shape and spreading evolution can be determined in a relatively straightforward manner. The Young-Laplace equation dictates that the droplet shape is part of a sphere represented by $r=R_{L}(z, t)$, where

$$
R_{L}^{2}(z, t)=\frac{a^{2}}{\cos ^{2}\left(\theta-\frac{\pi}{2}\right)}-\left(z-a \tan \left(\theta-\frac{\pi}{2}\right)\right)^{2} .
$$

Here $a(t)$ is the contact line position, $\theta(t)$ is the contact angle, $z$ is a vertical coordinate, and $r$ is a radial coordinate (see Figure 4.1a). The spherical geometry requires

$$
V_{L}=\frac{\pi}{3} \frac{a^{3}}{\sin ^{3} \theta}\left[2-3 \cos \theta+\cos ^{3} \theta\right]
$$

which relates $a(t)$ and $\theta(t)$ since the droplet volume $V_{L}$ is conserved during spreading.

One possible description of how such a drop evolves in time expresses the contact line speed as a function of the contact angle (e.g., Hoffman [41]). One such case has been considered by Ehrhard and Davis, who take

$$
\frac{d a}{d t}=\kappa\left(\theta-\theta_{A}\right)^{m}
$$

where $\kappa$ is a characteristic contact line speed, $\theta_{A}$ is an advancing contact angle, and $m$ is a spreading exponent. The parameters appearing in (3.3) in general depend on the specific liquid and substrate under consideration [16]. The parameter $\kappa$ is typically related to the velocity scale defined by $\gamma / \mu$ multiplied by additional modeling factors, as we note below. In the development of our model we shall interpret $\kappa$ as an independent parameter that represents a characteristic contact line speed. Equation (3.3) relates the apparent contact angle to the speed of the contact line and, in building in the micron-scale physics in this way, allows the contact line to be addressed on a 
macroscopic level. Such relationships have been examined by Dussan V. [2], Ehrhard and Davis [5], Ehrhard [16], among others. Glick [20] showed that a similar relationship, based on the work of McHale, Rowan, and Newton [42], coupled with the spherical cap approximation provided an accurate characterization of the spreading of polystyrene on a glass surface under isothermal conditions. In particular, McHale, Rowan, and Newton [42] used

$$
\frac{d a}{d t}=\frac{3 \gamma}{4 \mu J_{w}} \tan (\theta / 2)\left(\cos \theta_{A}-\cos \theta\right)
$$

where $J_{w}$ is a modeling factor associated with cutting off the viscous dissipation near the contact line. (Other contact line models have also been considered in the literature. In Hocking's model [7] the contact angle is assumed to be constant, but the meniscus shape is modified in the vicinity of the contact line so that a different apparent contact angle can be defined. The model of de Gennes [4] addresses the physics of a precursor film. Bertozzi et al. [43] use a mathematical condition of complete wetting to account for the contact line. Barenblatt, Beretta, and Bertsch [44] model the spreading problem by invoking an "autonomy principle" in a region between the bulk drop and the precursor film.)

In the limit of a thin drop with small capillary number in which the profile becomes approximately parabolic, Ehrhard and Davis [5] show that, for example, if $\theta_{A}=0$ in the above contact line condition (3.3), the axisymmetric drop evolution can be solved explicitly to find

$$
\frac{a(t)}{a_{0}}=\left[1+(3 m+1) \theta_{0}^{m} \frac{\kappa t}{a_{0}}\right]^{\frac{1}{3 m+1}},
$$

where $\theta_{0}$ is the initial contact angle and $a_{0}$ is the initial contact line position. The contact angle $\theta$ and the droplet shape are then also easily expressed explicitly in terms of $t$. Ehrhard [16] has performed experiments that support this simple model and provide values of $\kappa, \theta_{A}$, and $m$ for a given liquid on a given substrate. As can be observed in (3.5) if time is made dimensionless by $a_{0} / \kappa$, the dependence of the spreading dynamics on $\kappa$ can be scaled out of the problem so that the solutions are self-similar with respect to this parameter.

For a spherical drop with a large contact angle it may not be possible to write down explicit forms for $a$ and $\theta$ as functions of time, but their evolution is governed by a straightforward differential algebraic system of (3.2) and (3.3) which can be solved numerically. Such solutions are also self-similar with respect to $\kappa$.

The central idea here is that in the capillary-dominated limit (spherical cap approximation) and with the use of a contact line speed versus contact angle condition (3.3) the droplet shape evolution can be determined without many of the other complications in the problem (e.g., the fluid velocity is decoupled from the shape evolution problem). It is in this spirit that we shall develop the spreading and melting droplet model; that is, we shall identify an analogous simple model for the spreading and melting droplet configuration.

4. Spreading and melting: Hot plate configuration. The general problem of the spreading and melting droplet involves solving a thermal transport problem in the solid and liquid phases that accounts for phase transformation, and simultaneously solving the hydrodynamic equations of motion in the liquid phase, all of which is coupled with the free-boundary problem in which the solid-liquid interface, the 
liquid-vapor interface, the solid-vapor interface, the contact line, and the trijunction positions must be determined as part of the solution.

We would like to obtain from the general problem a model in which the freeboundary problem is effectively decoupled from the bulk hydrodynamics. We shall accomplish this by considering exclusively the physical limit of small capillary number $C \rightarrow 0$ in which surface tension forces control the shape of the liquid-vapor interface (Marangoni flows are not considered). In this limit, the momentum equation requires that the liquid pressure $p$ is spatially uniform and the normal-stress boundary condition on the liquid-vapor interface determines the liquid pressure in terms of the liquid-vapor interface curvature (this is the Young-Laplace condition). Since the pressure can depend only on time, the curvature is uniform in space and the liquid-vapor shape evolution is quasi-steady.

In conjunction with the small capillary number limit, we shall make the following simplifying assumptions regarding the thermal field and free-boundary problem. First, we shall assume that the thermal problem is diffusion-controlled so that convective effects are negligible. This decouples the hydrodynamic problem from the thermal problem except through the geometry. Second, we shall neglect gravity. Third, we shall assume that the solid-liquid phase boundary is planar and remains parallel to the substrate for all time so that its position $z=h(t)$ is a function of time only. As we shall see in section 4.2 this allows a simple description of the thermal problem but still retains a nonisothermal system that accounts for the change of phase.

The presence of phase-change in the spreading and melting droplet configuration gives rise to additional modeling issues not present in the isothermal spreading problem, in particular the treatment of the motion of solid phase and the trijunction.

An equation of motion for the solid [45] can be written as

$$
\frac{d}{d t} \vec{P}(t)=\sum_{i} \vec{F}_{i}
$$

where $\vec{P}(t)=M_{S}(t) \vec{V}_{C M}$ is the linear momentum of the solid, $M_{S}(t)$ is the mass of the solid, $\vec{V}_{C M}$ is the velocity of the center of mass of the solid, and $\vec{F}_{i}$ are the forces acting on the solid. Here we consider only symmetric droplets and therefore address only vertical motion of the solid. For the general spreading and melting droplet (in the absence of gravity) this equation of motion is (in dimensionless form)

$$
\begin{aligned}
\rho R e \frac{d}{d t}\left(M_{S}(t) \vec{V}_{C M}\right)= & -\frac{1}{C} \int_{\partial \Omega_{S L}} p \hat{n} d S+\int_{\partial \Omega_{S L}}\left[\nabla \vec{u}+(\nabla \vec{u})^{T}\right] \cdot \hat{n} d S \\
& +\frac{1}{C} \int_{t j} \sin (\pi-\phi)(-\hat{k}) d l,
\end{aligned}
$$

where $\rho$ is the density ratio (solid to liquid), $R e=\mathcal{R}_{0} \kappa / \nu$ is the Reynolds number, $\nu$ is the kinematic viscosity, $\vec{u}$ is the fluid velocity, $\phi$ is the angle through the liquid at the trijunction, and $\hat{k}$ is a unit vector in the $z$ direction (see Figure 4.1). Lengths have been made dimensionless with the initial radius of the spherical drop $\mathcal{R}_{0}$, time with $\mathcal{R}_{0} / \kappa$, velocities with $\kappa$, and pressure with $\gamma / \mathcal{R}_{0}$. Also, $\partial \Omega_{S L}$ represents the solidliquid interface and $t j$ represents the trijunction. Here all normal vectors are assumed to be outward from the solid. The forces accounted for in (4.2) are the pressure and viscous forces along the solid-liquid interface and surface tension forces at the trijunction. We have assumed that the force on the solid due to surface tension is $\gamma \sin (\pi-\phi)$ in the $-\hat{k}$ direction. Note that if we were to include gravitational effects, 

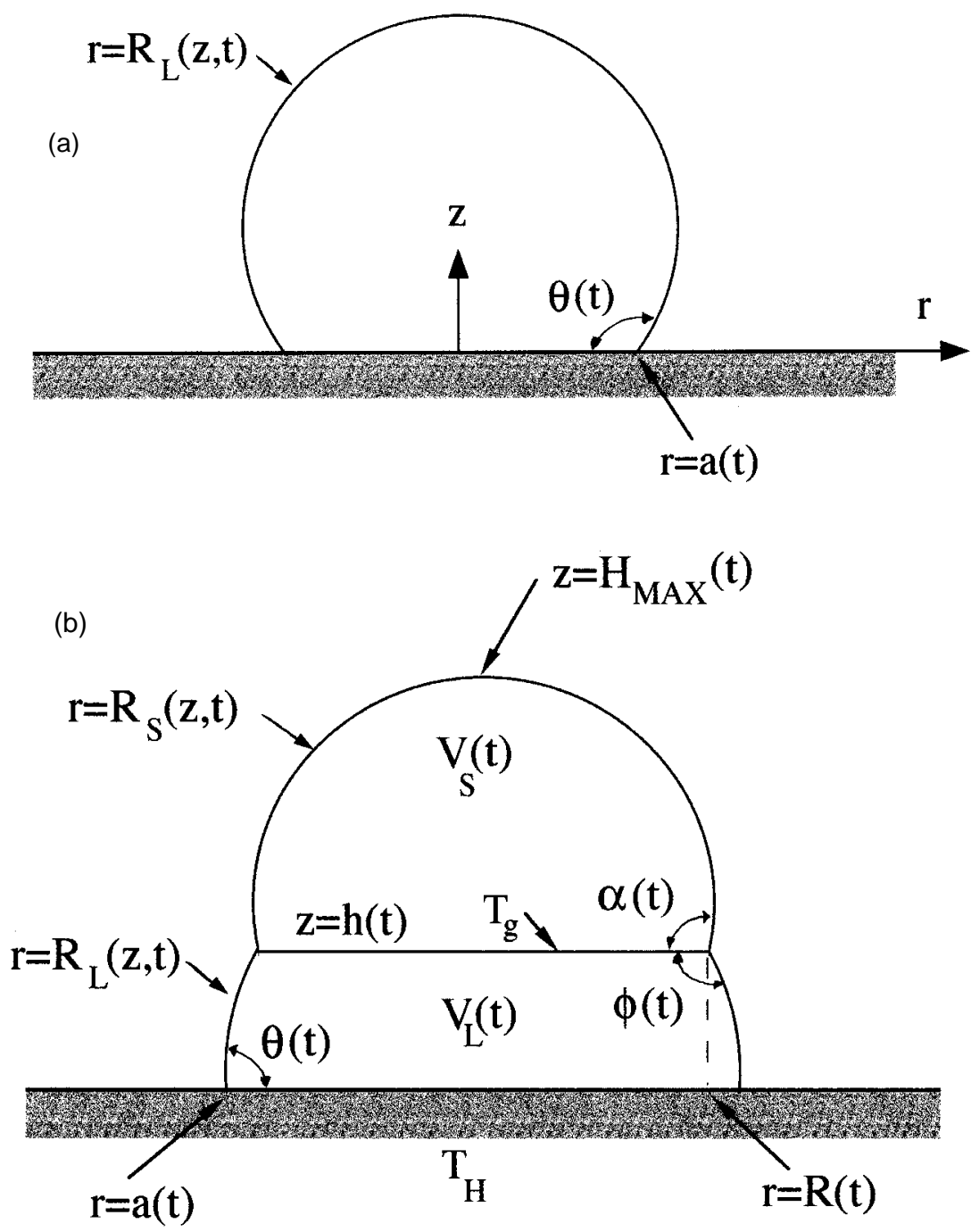

FIG. 4.1. In part (a) a sketch of the isothermal liquid droplet (oven) configuration is shown. The droplet is axisymmetric and evolves in time $t$. The vertical coordinate is $z$ and radial coordinate is $r$. The liquid-vapor interface is identified by $r=R_{L}(z, t)$. The radial position of the contact line is given by $r=a(t)$ and the contact angle is $\theta(t)$. The volume of this drop is constant in time. In part (b) a sketch of the spreading and melting droplet (hot plate) configuration is shown. The temperature of the hot plate is $T_{H}$ and the isotherm $T_{g}$ separates the solid and liquid phases $\left(T_{H}>T_{g}\right)$. Note that the liquid phase is between the hot plate and the solid portion of the droplet. The solid-liquid interface is assumed planar and is given by $z=h(t)$. The total droplet volume is divided between solid and liquid volumes, $V_{S}(t)$ and $V_{L}(t)$, respectively. The droplet is axisymmetric with solid-vapor interface given by $r=R_{S}(z, t)$ and liquid-vapor interface given by $r=R_{L}(z, t)$. The contact line is given by $r=a(t)$ and the associated contact angle is $\theta(t)$. The radial position of the trijunction is given by $r=R(t)$. The angle through the liquid phase at the trijunction is $\phi(t)$, while that through the solid phase is $\alpha(t)$. Finally, we identify the highest point of the drop by $z=H_{M A X}(t)$ 
there would be a force $M_{S}(t) g$ downward and a buoyancy force that would enter through the pressure integral. In the simplified model we consider, with $C \rightarrow 0$ and a planar solid-liquid interface, the equation of motion (4.2) reduces to its equilibrium form

$$
0=-\pi R^{2} p+2 \pi R \sin (\pi-\phi)
$$

where $R$ is the radial position of the trijunction.

We note that in solidification problems, it is common to impose at the trijunction a condition on the "growth angle," which is the angle between the tangents to the solid-vapor and liquid-vapor interfaces at the trijunction $[37,39,34,36]$. It is possible that some sort of growth angle, analogous to that for solidification, prevails at the trijunction here (perhaps it would be better termed a "melting" angle); however, the authors know of no experimental measurements of such an angle. We note that in the work of Surek and Coriell [35] on the float-zone problem, a growth angle condition was applied at the freezing front but not at the melting front. We shall find that in our model the equation of motion for the solid plays the role of the trijunction condition in a natural way in that it incorporates the capillary force associated with the trijunction and that it closes the mathematical model. Consequently, we shall refrain from imposing any additional conditions at the trijunction. The trijunction angle, in fact, comes out as part of the solution (Figure 4.4). We note that if the equation of motion is neglected in favor of a trijunction condition such as a fixed growth angle condition, the model fails to yield physical results (see section 5).

Based on the above assumptions, the hydrodynamic problem is decoupled from the thermal and shape (or free-boundary) problems. Below we describe the shape problem as it stands in the light of the above assumptions. A simple thermal model then couples to the shape problem only through the dynamics of $h(t)$.

4.1. The shape problem. We present the model in terms of dimensionless variables. Lengths are made dimensionless with the initial radius of the spherical drop $\mathcal{R}_{0}$, time with $\mathcal{R}_{0} / \kappa$ and pressure with $\gamma / \mathcal{R}_{0}$.

The unknown quantities that depend on space and time are the liquid-vapor interface position $r=R_{L}(z, t)$ and the solid-vapor interface position $r=R_{S}(z, t)$. Quantities that depend on time only are the maximum height of the droplet $H_{M A X}(t)$, the volume of the solid $V_{S}(t)$, the angle through the solid at the trijunction $\alpha(t)$, the radial position of the trijunction $R(t)$, the height of the solid-liquid interface $z=h(t)$, the volume of the liquid $V_{L}(t)$, the radial position of the contact line $a(t)$, the contact angle in the liquid at the substrate $\theta(t)$, the angle in the liquid at the trijunction $\phi(t)$, and the pressure in the liquid $p(t)$ (see Figure 4.1b).

We consider the evolution from an initially spherical drop given by

$$
R_{S}^{2}(z, 0)=1-[z+\cos \alpha(0)]^{2},
$$

where $\alpha(0)$ is the angle of contact the sphere makes with the substrate at time zero $(\alpha(0)=\pi$ for a perfect sphere). We can express the volume of the solid at any later time in terms of the angle $\alpha$ as

$$
V_{S}=\frac{\pi}{3}\left[2-3 \cos \alpha+\cos ^{3} \alpha\right]
$$

the trijunction position as

$$
R=\sin \alpha,
$$


and the maximum height as

$$
H_{M A X}=h+(1-\cos \alpha) .
$$

A global mass balance requires

$$
V_{L}=\frac{\pi}{3} \rho\left[-3 \cos \alpha(0)+\cos ^{3} \alpha(0)+3 \cos \alpha-\cos ^{3} \alpha\right] .
$$

The normal stress balance condition at the liquid-vapor interface gives $p(t)=$ $\nabla \cdot \hat{n}$, where the unit normal of the liquid-vapor interface $\hat{n}$ can be expressed as

$$
\hat{n}=\frac{\left(1,-R_{L z}\right)}{\sqrt{1+R_{L_{z}^{2}}^{2}}}
$$

and represents an equation for the shape of this interface. This equation is subject to the conditions at the contact line

$$
\begin{aligned}
R_{L}(0, t) & =a, \\
\frac{\partial R_{L}}{\partial z}(0, t) & =\tan \left(\theta-\frac{\pi}{2}\right)
\end{aligned}
$$

and also the conditions at the trijunction

$$
\begin{aligned}
R_{L}(h, t) & =R, \\
\frac{\partial R_{L}}{\partial z}(h, t) & =-\tan \left(\phi-\frac{\pi}{2}\right) .
\end{aligned}
$$

Furthermore, the pressure $p$, angle $\phi$, and position $R$ must conform to the equation of motion (4.3). It is worth noting that the general shape satisfying $p=\nabla \cdot \hat{n}$ is not necessarily one in which the liquid-vapor interface is spherical (it only requires constant curvature). However, the condition on pressure given by the equation of motion (4.3) in conjunction with conditions (4.10)-(4.13) does lead to a liquid-vapor interface that is part of a sphere given by

$$
R_{L}^{2}(z, t)=a^{2} \sec ^{2}\left(\theta-\frac{\pi}{2}\right)-\left[z-a \tan \left(\theta-\frac{\pi}{2}\right)\right]^{2},
$$

with

$$
\begin{gathered}
h=R \tan \left(\phi-\frac{\pi}{2}\right)+a \tan \left(\theta-\frac{\pi}{2}\right), \\
\frac{\sin \theta}{a}=\frac{\sin \phi}{R},
\end{gathered}
$$

and

$$
p=\frac{2 \sin \phi}{R} .
$$

Note that this spherical shape has a radius and a center that are not necessarily those of the initial solid shape. A further geometrical condition gives the liquid volume from the above shape in (4.14) as

$$
V_{L}=\frac{\pi}{3} \frac{a^{3}}{\sin ^{3} \theta}\left[-3 \cos \phi-3 \cos \theta+\cos ^{3} \phi+\cos ^{3} \theta\right] .
$$


Special considerations are required at the initial time and these are discussed in the context of our solution method. The above results complete the geometrical and mass balance laws that can be derived if surface tension controls the shape of the liquidvapor interface. Coupled with these geometrical considerations are a contact-line condition (3.3) and the dynamics of $h(t)$ which are determined through the thermal problem as described below.

4.2. Simple thermal model. In the above formulation we have assumed that the solid-liquid interface is planar and its position $z=h(t)$ depends only on time. The thermal problem determines $h(t)$ since this boundary is defined as the location of the solid-liquid interface temperature. In general, the thermal problem must include convective effects in the liquid; however, as mentioned above we neglect them here. We assume that the solid and liquid thermal properties are equal and the latent heat is zero. The use of zero latent heat is consistent with the idea of a glass transition between a highly viscous phase and a much less viscous phase as in Glick's experiments. For materials undergoing first-order phase transitions such as metals and alloys, where the effect of latent heat may be significant, this assumption may not be appropriate. We shall effectively treat the thermal problem for this system as that for a semi-infinite slab in which two-dimensional effects are neglected and the vapor phase is not distinguished from the droplet. With the above approximations in mind, we identify a standard similarity solution for the thermal field [46], described as follows.

Consider one-dimensional heat flow from a heated planar boundary of temperature $T_{H}>0$ and a far-field temperature of -1 , where temperatures are made dimensionless through

$$
T=\frac{T_{\text {dimensional }}-T_{g}}{T_{g}-T_{A}},
$$

where $T_{g}$ is the interface melting temperature and $T_{A}$ is the ambient far-field temperature in the vapor. The temperature throughout the droplet satisfies the heat equation

$$
P e \frac{\partial T}{\partial t}=\frac{\partial^{2} T}{\partial z^{2}},
$$

where $P e=\mathcal{R}_{0} \kappa / D^{t h}$ is the Peclet number and $D^{t h}$ is the thermal diffusivity. Equation (4.20) with the boundary conditions $T=T_{H}$ at $z=0$ and $T \rightarrow-1$ as $z \rightarrow \infty$ admits a similarity solution of the form

$$
T=T_{H}-\left(1+T_{H}\right) \frac{2}{\sqrt{\pi}} \int_{0}^{\eta} e^{-u^{2}} d u=T_{H}-\left(1+T_{H}\right) \operatorname{erf}(\eta)
$$

where $\operatorname{erf}(\eta)$ is the error function and $\eta$ is the similarity variable defined by

$$
\eta=\frac{z}{2 \sqrt{t / P e}} .
$$

We define $\eta_{g}$ as the value of $\eta$ corresponding to the solid-liquid interface temperature $T=0$ so that

$$
\operatorname{erf}\left(\eta_{g}\right)=\frac{T_{H}}{1+T_{H}}
$$


Therefore, the position of the solid-liquid interface is given by

$$
z=h(t)=2 \eta_{g} \sqrt{t / P e},
$$

corresponding to square-root in time growth.

We note that a much broader class of dynamics for the solid-liquid interface is possible. In addition to a nonplanar interface, it is also possible that the height of the front is nonmonotonic in time due to both thermodynamic and hydrodynamic effects. These more general shapes and dynamics are not considered here.

4.3. Geometrical model: Solution method. The ten equations (4.5), (4.6), (4.7), (4.8), (4.15), (4.16), (4.17), (4.18), (4.24), and (3.3) determine the ten unknowns $a(t), R(t), h(t), H_{M A X}(t), \theta(t), \phi(t), \alpha(t), p(t), V_{L}(t), V_{S}(t)$ and constitute the geometrical model. However, we can express the model in terms of a reduced set of variables $a(h), \alpha(h), \theta(h)$, and $\phi(h)$ with the independent variable $h$. These variables are governed by the differential algebraic system

$$
\begin{gathered}
\frac{d a}{d h}=\frac{1}{2} \mathcal{K}_{T}\left(\theta-\theta_{A}\right)^{m} h, \\
0=\sin \theta \sin \alpha-a \sin \phi, \\
0=\rho \sin ^{3} \theta\left[3 \cos \alpha-\cos ^{3} \alpha-3 \cos \alpha(0)+\cos ^{3} \alpha(0)\right] \\
-a^{3}\left[-3 \cos \phi-3 \cos \theta+\cos ^{3} \phi+\cos ^{3} \theta\right], \\
0=a \cos \phi+h \sin \theta+a \cos \theta .
\end{gathered}
$$

The remaining unknowns can be expressed in terms of these variables as

$$
p=\frac{2 \sin \theta}{a},
$$

$$
\begin{gathered}
V_{L}=\frac{\pi}{3} \frac{a^{3}}{\sin ^{3} \theta}\left[4-(1+\cos \theta)^{2}(2-\cos \theta)-(1+\cos \phi)^{2}(2-\cos \phi)\right], \\
V_{S}=\frac{\pi}{3}\left[4-(1+\cos \alpha)^{2}(2-\cos \alpha)\right] \\
R=\sin \alpha \\
H_{M A X}=h+(1-\cos \alpha), \\
t=\frac{1}{4} \mathcal{K}_{T} h^{2} .
\end{gathered}
$$

We have solved the above system (4.25)-(4.28) using the code DASSL [47].

The parameters appearing in this model are the spreading/heating parameter $\mathcal{K}_{T}$, the density ratio $\rho$, the advancing contact angle $\theta_{A}$, and the spreading exponent $m$. The first of these parameters is a combination of two others, namely, 
$\mathcal{K}_{T} \equiv P e / \eta_{g}^{2}=\left(\mathcal{R}_{0} \kappa\right) /\left(D^{t h} \eta_{g}^{2}\right)$. The parameter $\mathcal{K}_{T}$ measures the contact line spreading dynamics relative to the thermal dynamics of the phase transformation process. Consequently, if $\theta_{A}$ and $m$ are not temperature-dependent, for a given material on a given substrate only $\mathcal{K}_{T}$ changes as the substrate temperature changes. Small values of $\mathcal{K}_{T}$ correspond to slow spreading with relatively fast thermal dynamics and large values $\mathcal{K}_{T}$ correspond to rapid spreading with relatively slow thermal dynamics. As the results will show, large values of $\mathcal{K}_{T}$ lead to spreading dynamics that differ strongly from the isothermal spreading case since a greater percentage of the spreading occurs in the presence of the solid phase. When $\mathcal{K}_{T}$ is small, the spreading dynamics much more closely resemble that of isothermal spreading.

The initial conditions for the droplet evolution for the hot plate calculations are set up as follows. The equations are solved numerically by specifying an initial time $0<t_{0} \ll 1$ and the initial values of $h_{0}, a\left(h_{0}\right)$ and $\alpha\left(h_{0}\right), \theta\left(h_{0}\right)$, and $\phi\left(h_{0}\right)$ which follow from expanding the governing equations,

$$
\begin{aligned}
h\left(t_{0}\right) & =2 \sqrt{t_{0} / \mathcal{K}_{T}} \equiv h_{0}, \\
a\left(h_{0}\right) & =a(0)+\tilde{a} \\
\alpha\left(h_{0}\right) & =\alpha(0)-\tilde{\alpha} \\
\theta\left(h_{0}\right) & =\theta(0)-\tilde{\theta} \\
\phi\left(h_{0}\right) & =\phi(0)+\tilde{\phi}
\end{aligned}
$$

where for $a(0)=0, \alpha(0)=\pi, \theta(0)=\pi$, and $\phi(0)=0$ we have

$$
\begin{aligned}
& \tilde{a}=\frac{1}{4} \mathcal{K}_{T}\left(\theta(0)-\theta_{A}\right)^{m} h_{0}^{2}, \\
& \tilde{\alpha}=\sqrt{\frac{h_{0}}{\rho}+\frac{1}{\rho} \sqrt{h_{0}^{2}+2 \rho h_{0} \tilde{a}^{2}}}, \\
& \tilde{\theta}=\frac{2 h_{0}}{\tilde{a}} \frac{1}{(\tilde{\alpha} / \tilde{a})^{2}-1}, \\
& \tilde{\phi}=\frac{\tilde{\alpha} \tilde{\theta}}{\tilde{a}} .
\end{aligned}
$$

Here, $a(0), \alpha(0)$ are the true initial values for the initial solid shape (e.g., $a(0)=0$ and $\alpha(0)=\pi$ for a perfect sphere). We note that although $\theta(0)$ and $\phi(0)$ are technically not defined at $t=0$, we have taken $a(0)=R(0)=0, \theta(0)=\alpha(0)=\pi$, and $\phi(0)=0$ in these initial conditions and in the computations described below.

Given these initial conditions, (4.25)-(4.28) are integrated until the trijunction radius vanishes, $R\left(t_{F}\right)=0$, at some final time $t=t_{F}$. At that time, the droplet continues to evolve according to the oven (isothermal spreading) model (given by (3.2) and (3.3)) with initial conditions $a\left(t_{F}\right), \theta\left(t_{F}\right)$, and $V_{L}\left(t_{F}\right)$. We describe the results below.

4.4. Geometrical model: Results. In the hot plate cases, we shall use exclusively the contact line condition (3.3) where, unless otherwise specified, we have used $\theta_{A}=0.1 \pi, m=3$, and $\rho=1$. Figure 4.2 shows $\cos \theta$ versus the dimensionless time calculated for the hot plate cases shown in A-I in Table 4.1 and also the corresponding curve for the oven calculation. The solutions for the hot plate cases A-C nearly coincide with the oven result (these are the rightmost curves in the lower portion of the plot and the leftmost near the top). Increasing $\mathcal{K}_{T}$ corresponds to greater changes 


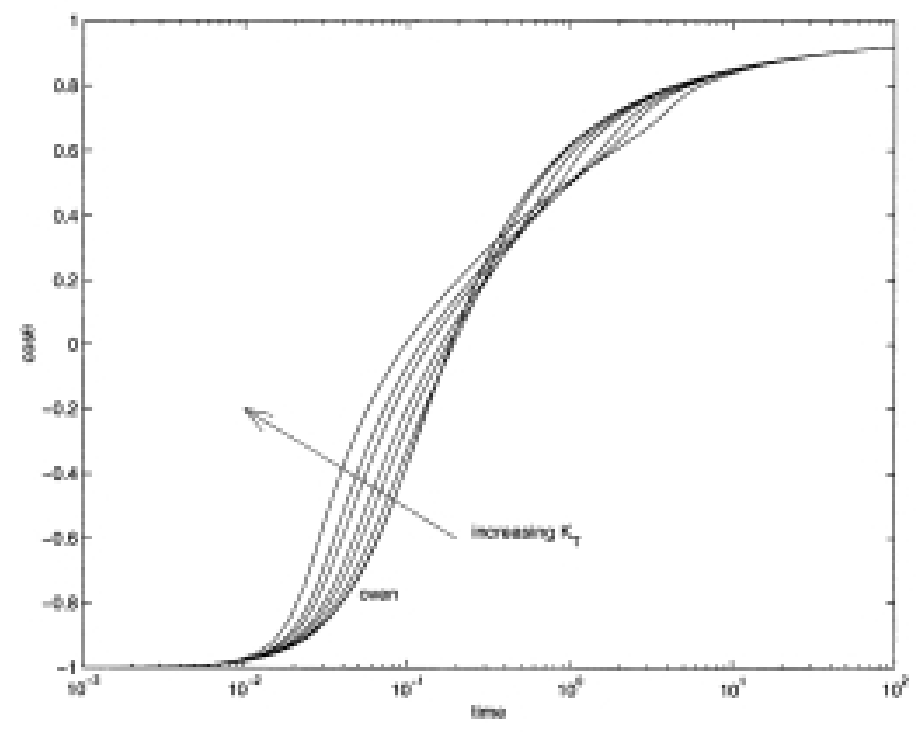

FIG. 4.2. This plot shows $\cos \theta(t)$ versus the dimensionless time calculated for the hot plate cases shown in $A-I$ in Table 4.1 and also the corresponding curve for the oven calculation. The solutions for the oven case (rightmost curve at the bottom and leftmost curve at the top) and the hot plate cases $A-C$ appear to nearly coincide. Only for cases $D-I$ ( $\mathcal{K}_{T}$ greater than or equal to unity) can a noticeable change be observed on this scale. Increasing $\mathcal{K}_{T}$ amplifies the difference between the oven and hot plate cases by bending the curve to the left near the bottom and to the right near the top, as shown.

TABLE 4.1

This table shows material parameters for the experiments of Glick [20] and the corresponding value of $\mathcal{K}_{T}$ calculated using $\kappa=\gamma / \mu$ and $D^{\text {th }}=0.8 \mathrm{~mm}^{2} / \mathrm{s}$. The values of $t_{F}$ are those predicted by the present model. In some of the figures we also show results for additional values of the dimensionless parameter $\mathcal{K}_{T}=5,10,15$, and 30, which we refer to as cases $F, G$, H, and I, respectively.

\begin{tabular}{|c|c|c|c|c|c|c|c|}
\hline & $T_{H}\left({ }^{\circ} \mathrm{C}\right)$ & $\eta_{g}$ & $\gamma(\mathrm{mN} / \mathrm{m})$ & $\mu(\mathrm{Pa} \cdot \mathrm{s})$ & $\gamma / \mu(\mathrm{mm} / \mathrm{s})$ & $\mathcal{K}_{T}$ & $t_{F}(\mathrm{~s})$ \\
\hline \hline $\mathrm{A}$ & 99 & 0.2761 & 36 & 45,000 & 0.0008 & 0.0131 & 15.9 \\
\hline $\mathrm{B}$ & 108 & 0.3456 & 35 & 8,300 & 0.004 & 0.0419 & 8.9 \\
\hline $\mathrm{C}$ & 118 & 0.4109 & 34 & 900 & 0.038 & 0.281 & 3.5 \\
\hline $\mathrm{D}$ & 131 & 0.4820 & 33 & 150 & 0.22 & 1.184 & 1.6 \\
\hline $\mathrm{E}$ & 138 & 0.5154 & 33 & 52 & 0.63 & 2.96 & 1.1 \\
\hline
\end{tabular}

in $\theta$ at early times; this trend is reversed for longer times. Note that there is not a universal curve characterizing the evolution for all temperatures for the hot plate configuration, as there is in the oven case.

Figure 4.3 shows the value of $a(t)$ corresponding to $\theta(t)$ in the previous figure. The leftmost curve in this figure shows the evolution of the oven case. For the hot plate evolution as $\mathcal{K}_{T}$ is increased, the curves shift to the right; as in the previous figure there is not a noticeable difference between the oven and hot plate cases on this scale until $\mathcal{K}_{T}$ is greater than unity. Figures 4.2 and 4.3 show together that the contact line becomes less mobile as $\mathcal{K}_{T}$ increases despite the fact that the contact angle, at least at early times, changes more rapidly. Consequently, the hot plate configuration leads to a less mobile contact line (wetting area) but a more "mobile" contact angle. 


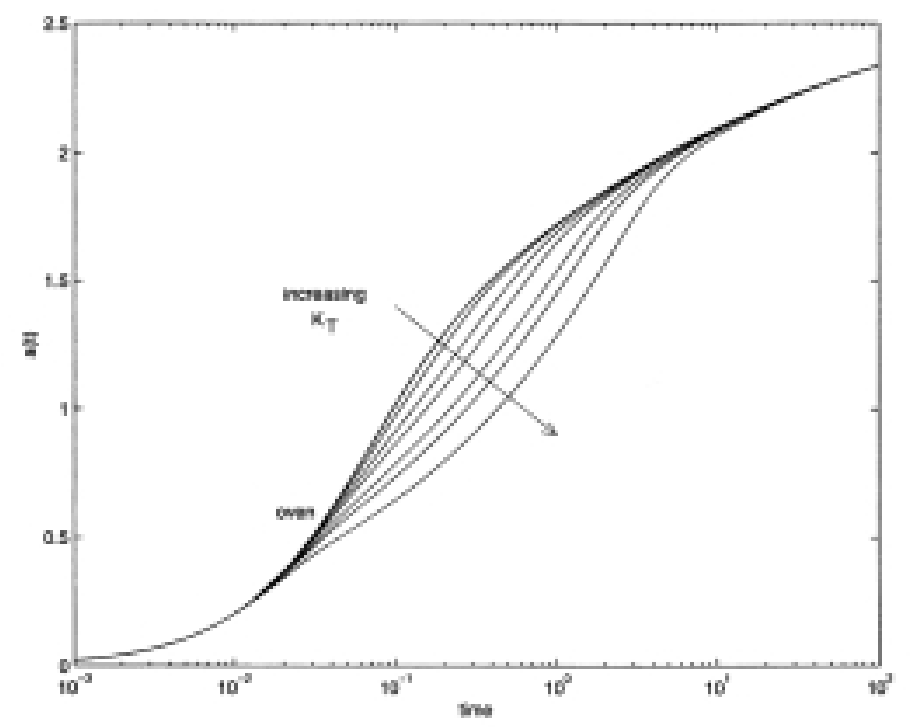

FIG. 4.3. This plot shows a(t) versus the dimensionless time calculated for the oven and hot plate cases shown in Figure 4.2. The oven case corresponds to the uppermost curve; increasing $\mathcal{K}_{T}$ moves the curve downwards, as shown. It is clear in this figure and Figure 4.2 that the solutions for the hot plate case are not self-similar with respect to a simple rescaling of time. Note that this figure shows that the contact line is less mobile as $\mathcal{K}_{T}$ increases despite the fact that the contact angle, at least initially, changes more rapidly.

Figure 4.4 shows the evolution of the angle between the solid-vapor interface and the liquid-vapor interface at the trijunction $\phi(t)+\alpha(t)-\pi$ for the hot plate cases F-I. We see several features from this plot. First, this angle starts out at zero, reaches a maximum, and then decreases monotonically to zero; the slope discontinuity at the trijunction varies dynamically. Second, the maximum amplitude of this quantity increases as $\mathcal{K}_{T}$ increases. Third, the time at which the droplet is completely melted varies with $\mathcal{K}_{T}$; this result is discussed in more detail in Figure 4.9. Note that the angle plotted in Figure 4.4 is not defined in the oven configuration.

Figure 4.5 compares $a(t), \theta(t), p(t)$, and $H_{M A X}(t)$ for the hot plate case I $\left(\mathcal{K}_{T}=\right.$ 30 ) and the oven case. As in the previous comparisons we see that the droplet in the hot plate case evolves more slowly with respect to the contact line position $a(t)$ than does the oven case (the presence of the solid inhibits spreading). On the other hand, at least in the initial stages, the contact angle departs from its initial value more quickly in the hot plate configuration. The pressure shows a peak in the hot plate configuration before a monotonic decline, while it declines monotonically for the oven case. Finally, the maximum height of the drop $H_{M A X}(t)$ decreases monotonically but has a noticeable change in slope around $t \approx 4$ where the solid disappears.

Figure 4.6 shows the variation of $R(t), V_{L}(t), \alpha(t)$, and $\phi(t)$ for the hot plate case I (see caption to Table 4.1). These quantities, with the exception of $V_{L}$, are not defined for the oven configuration. As is required for the spherical geometry, the trijunction position $R(t)$ begins at zero, reaches a maximum of unity, and then decreases to zero at the final time $t_{F} \approx 4$. The liquid volume increases monotonically in time, the solid angle $\alpha(t)$ decreases monotonically in time, and the liquid angle $\phi(t)$ at the trijunction increases monotonically from 0 to $\pi$. 


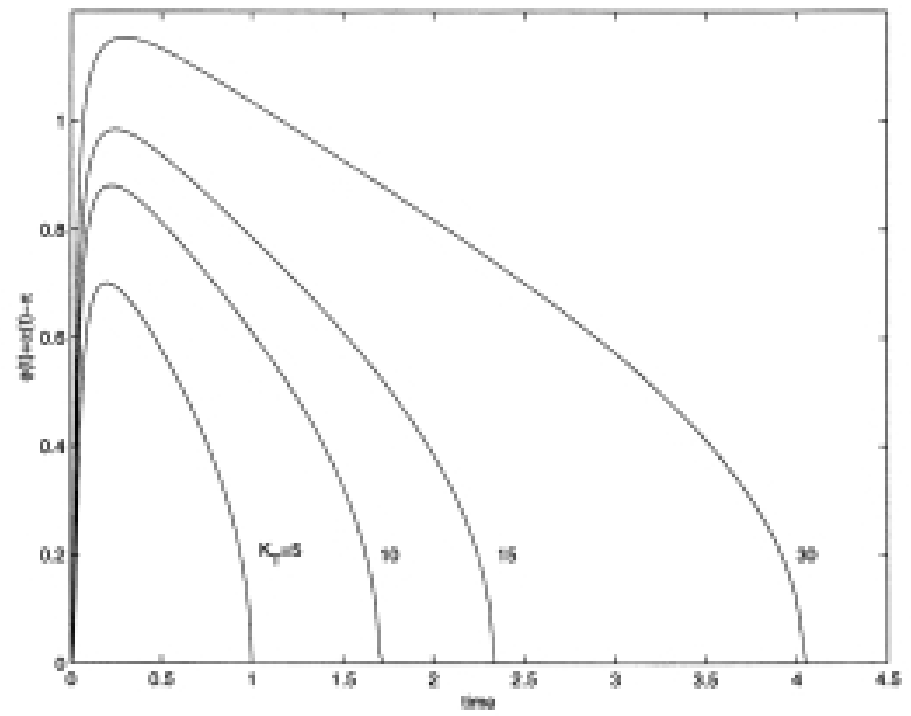

FIG. 4.4. This plot shows the variation of the angle $\phi(t)+\alpha(t)-\pi$ between the solid-vapor interface and the liquid-vapor interface at the trijunction versus the dimensionless time calculated for the hot plate case with the value of $\mathcal{K}_{T}$ given next to each curve. Nonzero values of $\phi(t)+\alpha(t)-\pi$ indicate a slope discontinuity at the trijunction.
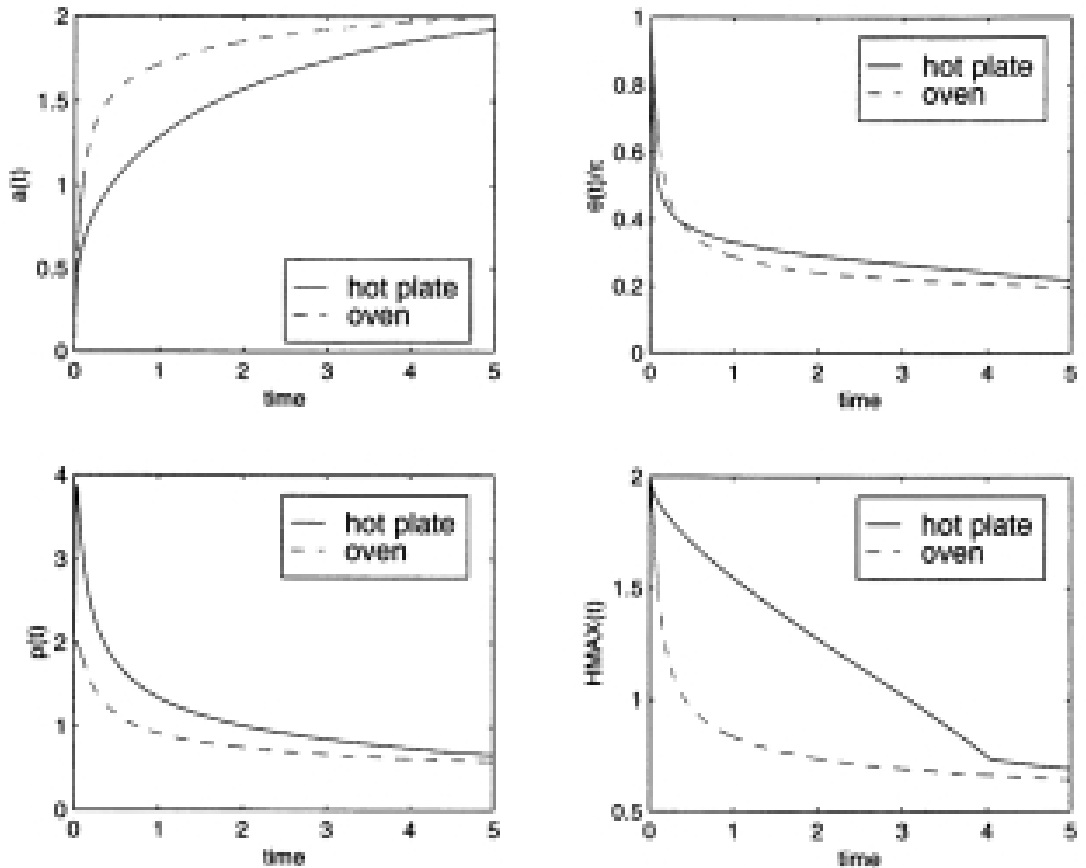

FIG. 4.5. This plot shows the contact line position $a(t)$, contact angle $\theta(t)$, pressure $p(t)$, and maximum height $H_{M A X}(t)$ versus the dimensionless time for the hot plate case $I\left(\mathcal{K}_{T}=30\right)$ and the oven case. The time at which the solid portion of the drop has completely disappeared is approximately $t_{F} \approx 4$. There is a noticeable change in the slope of $H_{M A X}(t)$ at this point. 

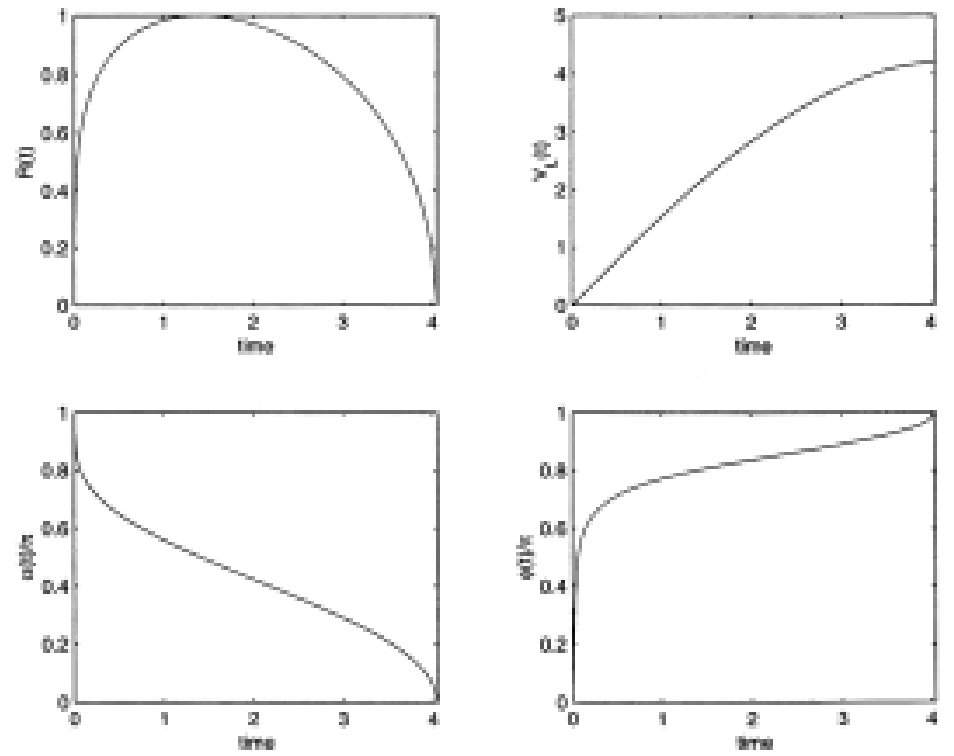

FIG. 4.6. This plot shows the trijunction position $R(t)$, the liquid volume $V_{L}(t)$, the solid angle $\alpha(t)$, and the liquid angle at the trijunction $\phi(t)$ versus the dimensionless time for the hot plate case $I\left(\mathcal{K}_{T}=30\right)$. Note that in the oven case, these variables, with the exception of the liquid volume which remains constant, are not defined.
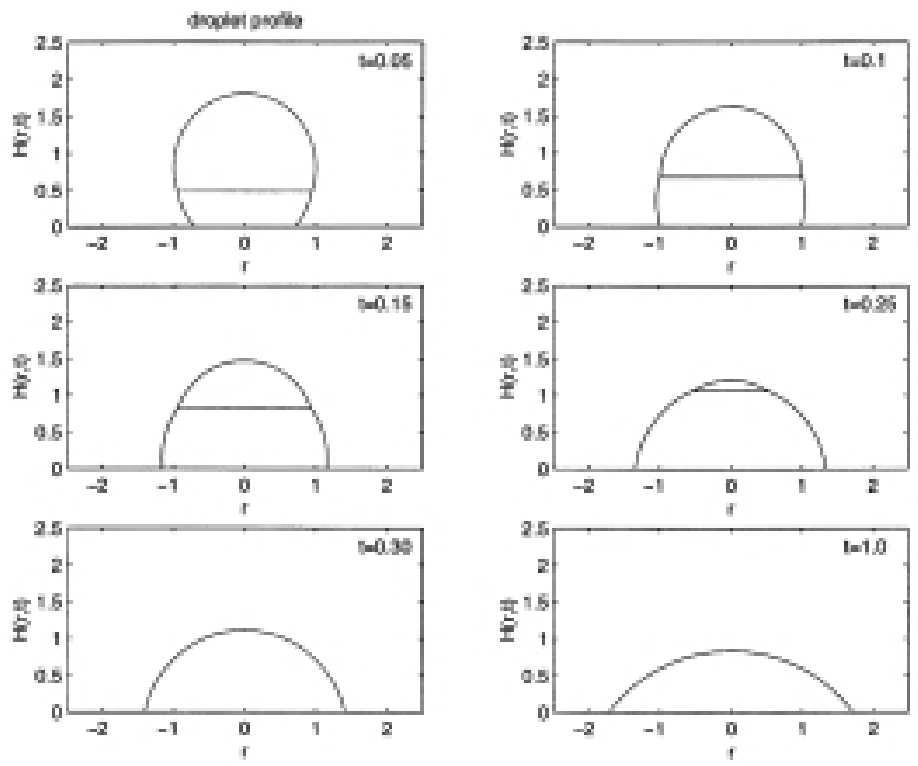

FIG. 4.7. This plot shows the shape evolution of the spreading and melting droplet as a function of time for $\mathcal{K}_{T}=0.884$. A comparison with Figure 4.8 shows qualitative differences between drop spreading with small and large values of $\mathcal{K}_{T}$.

Figures 4.7 and 4.8 show the shape evolution in the hot plate configuration for small $\mathcal{K}_{T}=0.884$ and large $\mathcal{K}_{T}=30$, respectively. In Figure 4.7 the spreading is 

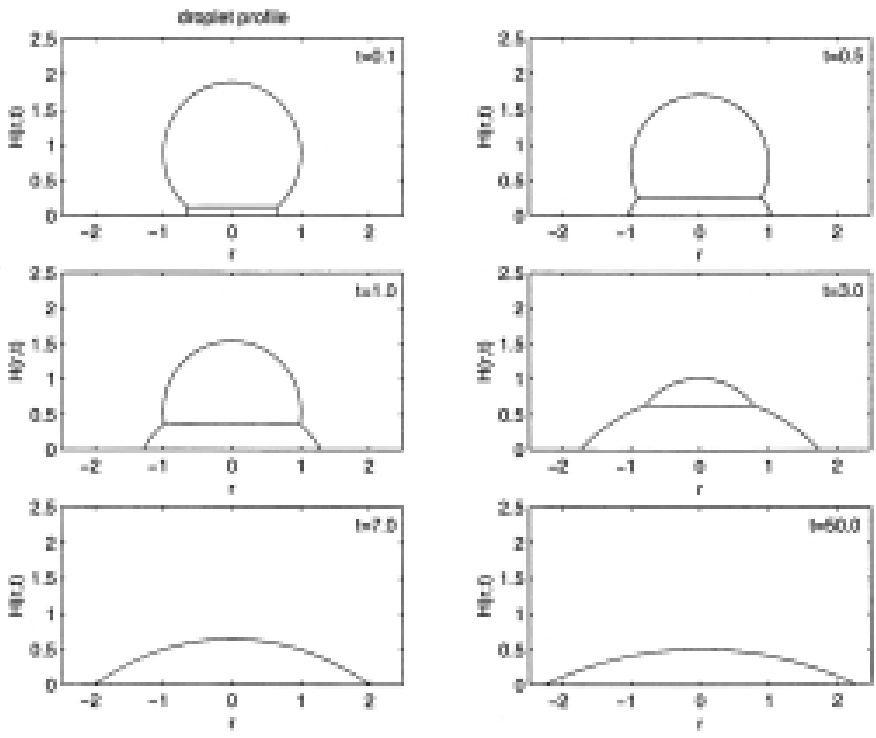

FIG. 4.8. This plot shows the shape evolution of the spreading and melting droplet as a function of time for $\mathcal{K}_{T}=30$.

relatively slow in comparison to the thermal dynamics of the melting drop. Note that if the spreading were stopped completely $\left(\mathcal{K}_{T}=0\right)$, then the profile would remain an exact sphere for all time and the melting front would simply pass through the motionless sphere. Figure $4.8\left(\mathcal{K}_{T}=30\right)$ shows that the liquid profile departs significantly from the original spherical shape. Note that at a fixed dimensionless time, say, $t=1$, the droplet in Figure 4.7 has completely melted and spread further than the droplet at the same time in Figure 4.8. However, the contact line position at the instant the solid disappears in Figure 4.8 is greater than that in Figure 4.7 , indicating that for larger $\mathcal{K}_{T}$ more spreading occurs in the presence of the solid phase.

In order to understand the effect of spreading on melting, we examine the disappearance time of the solid as $\mathcal{K}_{T}$ varies. First, we note that for the thermal field used in our model, $t=(h / 2)^{2} \mathcal{K}_{T}$. Therefore, if we imagine a horizontal isotherm passing from the bottom of a fixed sphere (of unit radius) to the top, the time it would take is $t_{F}=\mathcal{K}_{T}$ since the value of $h$ at the top of the sphere is 2. This result is shown by the dashed line in Figure 4.9. With this in mind, we examine the disappearance time of the solid for the hot plate case (where the sphere is spreading, not fixed) as $\mathcal{K}_{T}$ varies. Figure 4.9 shows that the dimensionless disappearance time (solid line) increases with increasing $\mathcal{K}_{T}$ but that the overall effect of spreading is to promote melting since these values are less than the corresponding ones represented by the line $t=\mathcal{K}_{T}$ for all $\mathcal{K}_{T}$. Note that the time scale used in the nondimensionalization of the time is $\mathcal{R}_{0} / \kappa$. For cases $\mathrm{A}-\mathrm{E}$ in Table 4.1 the predicted final dimensional times based on the velocity scale $\gamma / \mu$ are shown; these decrease with increasing temperature as expected. If one examines the dimensional final time as the parameter $\kappa$ varies with all other parameters held constant, the result is that the dimensional final time decreases with increasing $\kappa$, again indicating that spreading promotes melting. Our physical explanation of why spreading promotes melting in this model is that enhanc- 


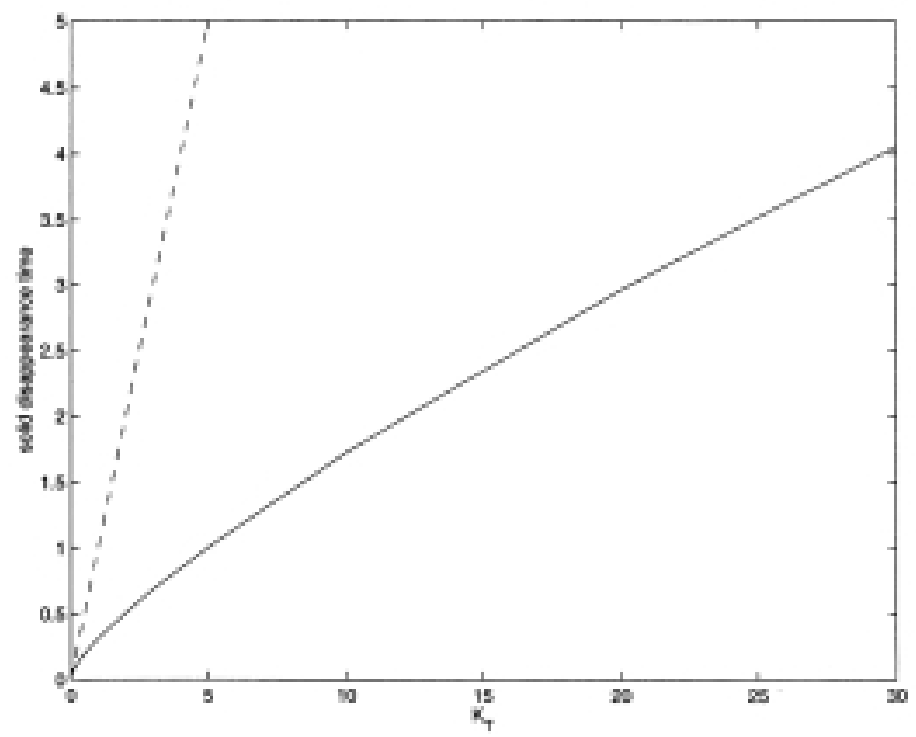

FIG. 4.9. This plot shows the dimensionless disappearance time for the solid versus $\mathcal{K}_{T}$ (solid line). Note that if the sphere were motionless and if one kept track of the position of the melting temperature isotherm, the time for it to reach the top of the drop would equal $\mathcal{K}_{T}$, since $t_{F}=$ $\left(h_{\text {top }} / 2\right)^{2} \mathcal{K}_{T}$ and $h_{\text {top }}=2$ in that case. This situation is shown by the dashed line. Therefore, since the actual $t_{F}$ is everywhere less than $\mathcal{K}_{T}$, we conclude that spreading promotes melting.

ing the spreading has the effect of bringing the remaining solid closer to the substrate where heating is more effective. The spreading effect does not modify the solid-liquid interface height $h(t)$, but it does modify the mass flux through this interface which is related to the velocity difference $h_{t}-h_{M A X t}$.

As described in section 3, the simple model for isothermal spreading allows the derivation of an explicit representation for the dynamics of $a(t)$. In the particular case of the Ehrhard and Davis [5] contact line model (3.3) the contact line position for a thin axisymmetric drop was found to follow the power law $a(t) \sim t^{1 /(3 m+1)}$. We do not have an analytical expression in the spreading and melting case but can gain insight on the general scaling properties by examining the numerical solutions.

Figure 4.10 shows a $\log -\log$ plot of $a(t)$ versus $t$ for the oven case and several hot plate cases as $\mathcal{K}_{T}$ is varied. Here we have taken $\rho=1, \theta_{A}=0$, and $m=3$. In this figure, the uppermost solid curve corresponds to the oven case. The curves for the hot plate case deviate from this curve as $\mathcal{K}_{T}$ increases and display at least three distinct regions. The dashed line on the left corresponds to a slope of unity indicating linear growth at early times. The dashed line on the right has a slope of $1 /(3 m+1)=1 / 10$, corresponding to that of Ehrhard and Davis for a thin isothermal liquid droplet. At intermediate times, where the droplet is still spreading and melting, there is an overall trend between a linear scaling and the 1/10 scaling of the isothermally spreading liquid drop. Although the evolution does not fit a specific power law here, a scaling behavior of $t^{1 / 3}$ is an approximate fit to the overall trend. We note that this scaling is a function of the exponent $m$, as shown in Figure 4.11.

Figure 4.11 shows a $\log -\log$ plot of $a(t)$ versus $t$ with fixed $\mathcal{K}_{T}=45, \rho=1$, $\theta_{A}=0$ and different values for $m$. The initial region maintains a linear scaling for sufficiently small times, but the intermediate and long time scalings both change with 


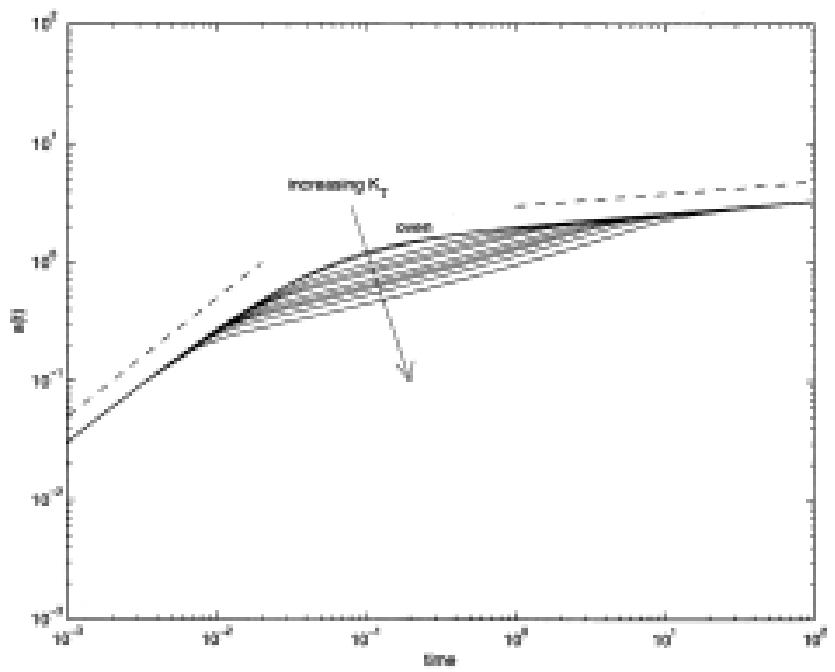

FIG. 4.10. This plot shows the contact line position a $(t)$ versus dimensionless time for the oven case and for a number of hot plate cases as $\mathcal{K}_{T}$ varies $\left(m=3, \theta_{A}=0\right)$. The uppermost solid curve corresponds to the oven case, as indicated. Results for the hot plate case (values of $\mathcal{K}_{T}$ used are $2.96,5.0,10.0,15.0,30.0,45.0,60.0,100.0,200.0)$ deviate from the oven case as shown. The dashed line on the left corresponds to a slope of unity, indicating that a $(t)$ initially grows linearly in time. The dashed line on the right has a slope of $1 /(3 m+1)=1 / 10$ which corresponds to that of Ehrhard and Davis for a thin isothermal drop and applies here when the droplet has completely melted and is thin enough. At intermediate times, where the droplet is still spreading and melting, there is an overall trend that is slower than linear but faster than the 1/10 scaling; however, this evolution does not appear to follow a specific power law.

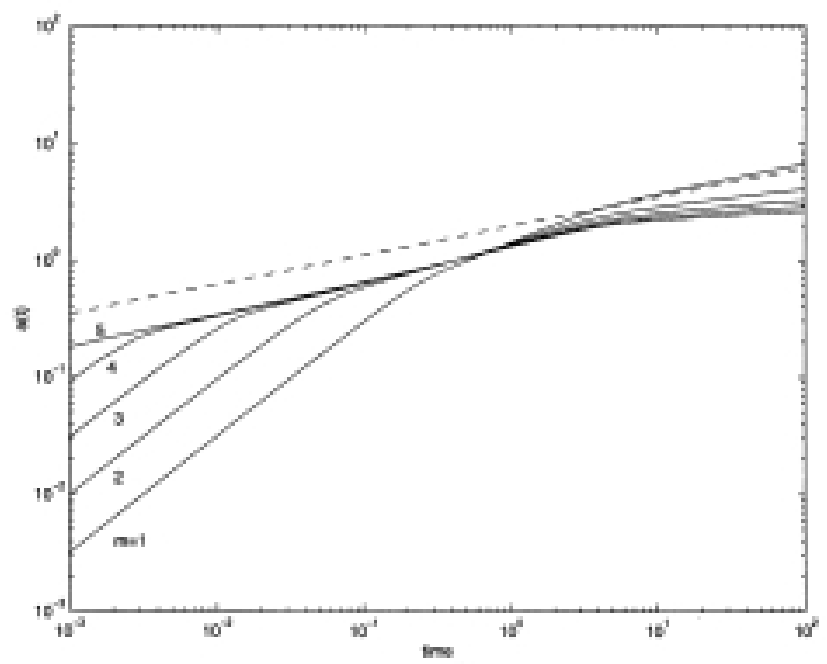

FIG. 4.11. This plot shows the contact line position a $(t)$ versus dimensionless time for the hot plate case with $\mathcal{K}_{T}=45, \theta_{A}=0$, and different values for $m$ as shown next to each curve. The dashed line has slope $1 / 4$ which seems to agree with the intermediate time evolution for the larger values of $m$. It can also be seen that the case for $m=1$ approaches a $t^{1 / 4}$ scaling for long times, when the drop is completely liquid and thin. 
$m$. The dashed line has slope $1 / 4$, which matches the intermediate time evolution for the larger values of $m$. It can also be seen that when $m=1$, or $1 /(3 m+1)=1 / 4$, the dynamics of $a(t)$ approach a $t^{1 / 4}$ scaling for long times, when the drop is completely liquid and thin.

5. Discussion. As mentioned earlier, our modeling has been motivated by the hot plate and oven experiments of Glick [20]. One point of comparison between the experimental results and the predictions of the present model are the temperaturedependent length scale $R_{\text {eff }}$ data identified by Glick in the hot plate case. In his work, it was observed that if a temperature-dependent length scale was introduced, the contact angle versus time data for the hot plate case collapsed to a master curve. This temperature-dependent length scale increased with increasing substrate temperature. Since the contact line model used by Glick (see (3.4)) had as the characteristic contact line velocity the temperature-dependent quantity $\gamma(T) / \mu(T)$ the need for additional temperature-dependence suggested another length scale in the problem not already taken into account by the isothermal (oven) spreading model.

There is no suggestion in our predictions that the solution is self-similar with respect to the parameter $\mathcal{K}_{T}$ in the hot plate case. However, there are a number of length scales that can be identified in our model, and we have shown these in comparison with the values of $R_{\text {eff }}$ obtained by Glick in Table 5.1 as they vary with the single parameter $\mathcal{K}_{T}$. The results of Table 5.1 show in dimensionless terms the final time $t_{F}$ where the solid disappears and the associated values of the contact line position $a\left(t_{F}\right)$ and the droplet height $H_{M A X}\left(t_{F}\right)$. The quantity $a^{\star}$ has been identified as the value of $a$ at which the hot plate dynamics first deviate noticeably from the oven dynamics as observed in Figure 4.10. There is an associated time $t^{\star}$, trijunction radius $R^{\star}$, and measures of the liquid volume $\left(3 V_{L}^{\star} / 4 \pi\right)^{1 / 3}$ and the solid volume $\left(3 V_{S}^{\star} / 4 \pi\right)^{1 / 3}$ (shown in parentheses in the last column). The significance of this point in time is that it appears to be the onset of the dynamics characterized by simultaneous spreading and melting.

We see that there is no noticeable difference between the hot plate and oven predictions for small values of $\mathcal{K}_{T}$ (and so no $a^{\star}$ is identified). The general trends in all the length scales shown with the exception of $a\left(t_{F}\right)$ and $\left(3 V_{S}^{\star} / 4 \pi\right)^{1 / 3}$ indicate the opposite trend of that observed by Glick (see Table 5.1). The solid volume measure $\left(3 V_{S}^{\star} / 4 \pi\right)^{1 / 3}$ may be in the general range of $R_{e f f}(T)$, indicating that $R_{e f f}(T)$ may be related to a length scale associated with the solid, but identification of this quantity is not clear for the smaller values of $\mathcal{K}_{T}$ where the experiments were performed.

Part of the difference between Glick's data and the present theory may be associated with the interpretation of $\mathcal{K}_{T}$ and its dependence on temperature. Recall that this parameter is defined as $\mathcal{K}_{T}=\left(\mathcal{R}_{0} \kappa\right) /\left(D^{t h} \eta_{g}^{2}\right)$. The parameter $\eta_{g}$ is an increasing function of $T$ and so if this were the only temperature-dependent quantity in this parameter group, then $\mathcal{K}_{T}$ would decrease with increasing temperature. However, if one interprets $\kappa=\gamma / \mu$ as the characteristic spreading velocity, then there is an additional temperature dependence, which, as indicated by Glick's data in Table 4.1, causes $\mathcal{K}_{T}$ to increase with increasing temperature. In the present model for spreading and melting, as $\mathcal{K}_{T} \rightarrow 0$ the hot plate dynamics approach that of the oven configuration since small $\mathcal{K}_{T}$ means that very little spreading occurs before the droplet is completely melted. Glick's interpretation of the temperaturedependent length scale, on the other hand, noted that $R_{\text {eff }}$ approached the radius of the original sphere $\mathcal{R}_{0}$ as the temperature approached infinity (that is, $R_{\text {eff }}=\mathcal{R}_{0}$ 
TABLE 5.1

The values for $R_{\text {eff }}$ are from Glick [20]. All lengths in the table are given in units of $\mathcal{R}_{0}$ and times are given in units of $\mathcal{R}_{0} / \kappa$. Here $\rho=1, \theta_{A}=0$, and $m=3$. The starred quantities represent the hot plate spreading case at the moment the dynamics of a $(t)$ deviate noticeably from that in the oven case (e.g., see Figure 4.10). In the final column the quantity in parentheses shows $\left(3 V_{S}^{\star} / 4 \pi\right)^{1 / 3}$. For the first three cases shown here there is no significant difference between the oven and hot plate results. The estimates involving the starred quantities are only approximate and only increasing or decreasing trends should be taken from this information.

\begin{tabular}{|c||c||c|c|c|c|c|c|c|}
\hline $\mathcal{K}_{T}$ & $R_{e f f}$ & $t_{F}$ & $a\left(t_{F}\right)$ & $H_{M A X}\left(t_{F}\right)$ & $t^{\star}$ & $a^{\star}$ & $R^{\star}$ & $\left(3 V_{L}^{\star} / 4 \pi\right)^{1 / 3}$ \\
\hline \hline 0.0131 & 0.2 & 0.0124 & 0.326 & 1.95 & - & - & - & - \\
\hline 0.0419 & 0.2 & 0.0328 & 0.684 & 1.77 & - & - & - & - \\
\hline 0.281 & 0.5 & 0.112 & 1.26 & 1.26 & - & - & - & - \\
\hline 2.96 & 0.8 & 0.526 & 1.71 & 0.843 & 0.03 & 0.6 & 0.9 & $0.5(0.9)$ \\
\hline 10 & - & 1.26 & 1.90 & 0.710 & 0.01 & 0.4 & 0.5 & $0.2(1)$ \\
\hline 30 & - & 2.77 & 2.05 & 0.614 & 0.01 & 0.3 & 0.4 & $0.1(1)$ \\
\hline 60 & - & 4.70 & 2.16 & 0.557 & 0.009 & 0.2 & 0.3 & $0.1(1)$ \\
\hline 100 & - & 6.76 & 2.24 & 0.520 & 0.008 & 0.2 & 0.3 & $0.09(1)$ \\
\hline 200 & - & 11.3 & 2.35 & 0.476 & 0.007 & 0.2 & 0.2 & $0.06(1)$ \\
\hline
\end{tabular}

if the substrate temperature is high enough to liquify the drop rapidly relatively to the spreading effect). Therefore, Glick's data suggests taking $\mathcal{K}_{T} \rightarrow \infty$ to recover the oven scaling $R_{\text {eff }} \rightarrow \mathcal{R}_{0}$, while the model suggests $\mathcal{K}_{T} \rightarrow 0$ in order to recover the oven model predictions. Consequently, we cannot identify with confidence a temperature-dependent length scale that is consistent with the one identified by Glick.

We note that even with a length scale that behaved perfectly consistently to the $R_{e f f}$ identified by Glick, our contact angle versus time data is not self-similar in that the shape of the curve $\cos \theta$ versus $t$ (e.g., see Figure 4.2) varies with the parameter $\mathcal{K}_{T}$ and therefore does not collapse to a single master curve with a rescaling of time.

A second point of discussion is that in our preliminary work on this problem we had developed a geometrical spreading and melting model as described above in the thin drop limit, where instead of imposing the equation of motion for the solid we considered an independent relationship at the trijunction. Specifically, drawing an analogy with the work of Anderson, Worster, and Davis [36], we imposed a condition that the tangents of the solid-vapor interface and the liquid-vapor interface at the trijunction met there with the same slope - the "zero growth angle" condition. We found, however, upon solving this slightly different system that a nonphysical result was obtained. Specifically, the melting droplet evolved in such a way that required a reversal in time in order for the trijunction position $R(t)$ to reach zero and for the solid to disappear. In the results of the model described in this paper where we have taken into account the equation of motion for the solid, we have shown that the tangents to the solid-vapor and liquid-vapor interfaces at the trijunction do not meet at an angle that stays fixed in time (see Figure 4.4). This can also be easily observed in the droplet profiles in Figures 4.7 and 4.8. We also point out that the zero growth angle condition does not in general require that the pressure in the liquid be positive and that a negative pressure corresponds to a concave up (or concave out) interface. In contrast, the trijunction condition derived from the equation of motion requires a 
positive liquid pressure to supply a force which balances the downward capillary force on the solid at the trijunction.

Finally, the equation of motion is a central ingredient for this spreading and melting droplet geometry. Our simplified model incorporates the equilibrium version of this condition. With the present thermal model, the solid-liquid interface advances monotonically in time as $t^{1 / 2}$. However, it is quite possible in reality that the motion of the solid-liquid interface is nonmonotonic. The solid-liquid interface could remain at a constant level if, for example, the solid was massive enough to create a squeeze film of constant thickness as the solid melted. Alternatively a suction-type of hydrodynamic effect, perhaps due to rapid advance of the contact line, could reduce the height of the solid-liquid interface during melting. Such effects are beyond the scope of the present work, but the geometrical model developed here may provide a basis upon which to begin to address these situations.

6. Conclusions. We have derived a simple model which describes the simultaneous spreading and melting of an initially spherical solid droplet. The model contains a moving contact line, a moving trijunction, and an internal solid-liquid boundary in a nonisothermal setting. A key physical parameter in this system is $\mathcal{K}_{T}=\left(\mathcal{R}_{0} \kappa\right) /\left(D^{t h} \eta_{g}^{2}\right)$, where $\mathcal{R}_{0}$ is the initial radius of the spherical drop, $\kappa$ is the characteristic contact line velocity, $D^{\text {th }}$ is the thermal diffusivity of the material (assumed equal in both solid and liquid phases), and $\eta_{g}$ is a thermal parameter that accounts for the temperature of the hot plate. The only other parameters in the model are the advancing contact angle $\theta_{A}$, the spreading exponent $m$ (see (3.3)) and the density ratio $\rho=\rho_{S} / \rho_{L}$.

The model has been derived around the following assumptions. (1) The initial solid droplet shape is a perfect sphere. (2) The liquid phase, once formed, is capillarydominated so that the liquid-vapor interface has constant curvature (the solution shows that it is in fact part of a sphere). (3) Gravity is negligible. (4) The melting droplet is axisymmetric so that the solid portion of the drop moves only vertically. (5) The thermal field is controlled by vertical diffusion only (there is no transverse heat loss and no convective heat transfer) and there is no latent heat release at the solidliquid interface, which is taken to be planar. Thermal properties are asssumed to be constant and equal in both phases. (6) We adopt a standard phenomenology for the contact line dynamics and keep this relation the same for both the oven (isothermal spreading) and hot plate (spreading and melting) configurations as a control. In this physical regime, spreading and melting are characterized by the single parameter $\mathcal{K}_{T}$. This observation means that our model isolates the spreading and melting interactions with all other physics controlled, albeit simplified.

The main conclusions we find are the following. First, the difference between the oven case (isothermal liquid spreading) and the hot plate case (spreading and simultaneous melting) is amplified as the parameter $\mathcal{K}_{T}$ is increased. Large values of $\mathcal{K}_{T}$ correspond to a relatively strong (or fast) contact line dynamics and a relatively weak (or slow) phase change dynamics. Second, the contact line in the spreading and melting configuration is less mobile than that in the isothermal liquid spreading configuration (the presence of the solid inhibits spreading relative to a completely liquid droplet). Third, the contact angle in the spreading and melting configuration tends to relax faster initially compared to the isothermal liquid spreading case. Finally, if the spreading effect is increased with all other thermal controls held fixed, the time for the solid to completely melt is decreased (spreading promotes melting). 
Acknowledgments. The authors would like to thank W.J. Boettinger, M. Rubinstein, T.J. Singler, P.H. Steen, and M.G. Worster for helpful conversations.

\section{REFERENCES}

[1] E.B. Dussan V. AND S.H. Davis, On the motion of a fluid-fluid interface along a solid surface, J. Fluid Mech., 65 (1974), pp. 71-95.

[2] E.B. Dussan V., On the spreading of liquids on solid surfaces: Static and dynamic contact lines, Annu. Rev. Fluid Mech., 11 (1979), pp. 371-400.

[3] S.H. DAvis, Contact-line problems in fluid mechanics, J. Appl. Mech., 50 (1983), pp. 977-982.

[4] P.G. De Gennes, Wetting: Statics and dynamics, Rev. Modern Phys., 57 (1985), pp. 827-863.

[5] P. Ehrhard And S.H. Davis, Non-isothermal spreading of liquid drops on horizontal surfaces, J. Fluid Mech., 229 (1991), pp. 365-388.

[6] P.J. Haley and M.J. Miksis, The effect of the contact line on droplet spreading, J. Fluid Mech., 223 (1991), pp. 57-81.

[7] L.M. Hocking, Rival contact angle models and the spreading of drops, J. Fluid Mech., 239 (1992), pp. 671-681.

[8] Y.D. Shikhmurzaev, Moving contact lines in liquid/liquid/solid systems, J. Fluid Mech., 334 (1997), pp. 211-249.

[9] G.J. Merchant and J.B. Keller, Contact angles, Phys. Fluids A, 4 (1992), pp. 477-485.

[10] P.A. Thompson And M.O. RobBins, Simulations of contact-line mobility: Slip and dynamic contact angle, Phys. Rev. Lett., 63 (1989), pp. 766-769.

[11] P.A. Thompson, W.B. Brinckerhoff, And M.O. Robbins, Microscopic studies of static and dynamic contact angles, J. Adhesion Sci. Technol., 7 (1993), pp. 535-554.

[12] J. Koplik and J.R. Banavar, Continuum deductions from molecular hydrodynamics, Annu. Rev. Fluid Mech., 27 (1995), pp. 257-292.

[13] D. JACQmin, An energy approach to the continuum surface tension method, in Proceedings of the 34th Aerospace Sciences Meeting and Exhibit, AIAA paper 96-0858, Reno, NV, 1996.

[14] D. JaCQmin, Contact-line dynamics of a diffuse fluid interface, J. Fluid Mech., 402 (2000), pp. $57-88$.

[15] P. Seppecher, Moving contact lines in the Cahn-Hilliard theory, Internat. J. Engrg. Sci., 34 (1996), pp. 977-992.

[16] P. Ehrhard, Experiments on isothermal and non-isothermal spreading, J. Fluid Mech., 257 (1993), pp. 463-483.

[17] J.A. Marsh, S. Garoff, and E.B. Dussan V., Dynamic contact angles and hydrodynamics near a moving contact line, Phys. Rev. Lett., 70 (1993), pp. 2778-2781.

[18] Q. Chen, E. Ramé, And S. GarofF, The velocity field near moving contact lines, J. Fluid Mech., 337 (1997), pp. 49-66.

[19] P. Thiansathaporn, Polymer Spreading at the Nanometer Scale, Ph.D. thesis, University of North Carolina, Chapel Hill, NC, 1998.

[20] D.D. GLick, Optical and Scanning Probe Microscope Studies of Polymer Spreading, Ph.D. thesis, University of North Carolina, Chapel Hill, NC, 1998.

[21] M.K. Sмітн, Thermocapillary migration of a two-dimensional liquid droplet on a solid surface, J. Fluid Mech., 294 (1995), pp. 209-230.

[22] S. Moosman And G.M. Homsy, Evaporating menisci of wetting fluids, J. Coll. Int. Sci., 73 (1980), pp. 212-223.

[23] P.C. WAYNER, JR., Spreading of a liquid film with a finite contact angle by the evaporation/condensation process, Langmuir, 9 (1993), pp. 294-299.

[24] D.M. Anderson And S.H. Davis, The spreading of volatile liquid droplets on heated surfaces, Phys. Fluids, 7 (1995), pp. 248-265.

[25] L.M. Hocking, On contact angles in evaporating liquids, Phys. Fluids, 7 (1995), pp. 2950-2955.

[26] S.J.S. Morris AND V. Moreno, Systematic analysis of an evaporating wetting meniscus on a smooth surface, in Proceedings of the ASME National Heat Transfer Conference, 1997, pp. 51-59.

[27] C.L. Tien, Fluid mechanics of heat pipes, Annu. Rev. Fluid Mech., 7 (1975), pp. 167-185.

[28] R.J. Braun, B.T. Murray, W.J. Boettinger, and G.B. McFadden, Lubrication theory for reactive spreading of a thin drop, Phys. Fluids, 7 (1995), pp. 1797-1810.

[29] J.A. Warren, W.J. Boettinger, and A.R. Roosen, Modeling reactive wetting, Acta Materialia, 46 (1998), pp. 3247-3264.

[30] S.H. Davis AND L.M. Hocking, Spreading and imbibition of viscous liquid on a porous base, Phys. Fluids, 11 (1999), pp. 48-57. 
[31] S.H. Davis ANd L.M. Hocking, Spreading and imbibition of viscous liquid on a porous base. II, Phys. Fluids, 12 (2000), pp. 1646-1655.

[32] S. Schiaffino AND A.A. Sonin, Motion and arrest of a molten contact line on a cold surface: An experimental study, Phys. Fluids, 9 (1997), pp. 2217-2226.

[33] S. Schiaffino And A.A. Sonin, Molten droplet deposition and solidification at low Weber numbers, Phys. Fluids, 11 (1997), pp. 3172-3187.

[34] R.A. Brown, Theory of transport processes in single crystal growth from the melt, AIChE J., 34 (1988), pp. 881-911.

[35] T. Surek And S.R. Coriell, Shape stability in float zoning of silicon crystals, J. Crystal Growth, 37 (1977), pp. 253-271.

[36] D.M. Anderson, M.G. Worster, and S.H. Davis, The case for a dynamic contact angle in containerless solidification, J. Crystal Growth, 163 (1996), pp. 329-338.

[37] T. Surek And B. Chalmers, The direction of growth of the surface of a crystal in contact with its melt, J. Crystal Growth, 29 (1975), pp. 1-11.

[38] G.A. Satunkin, V.A. Tatarchenko, and V.I. Shaitanov, Determination of the growth angle from the shape of a crystal lateral face and solidified separated drops, J. Crystal Growth, 50 (1980), pp. 133-139.

[39] A. SANZ, The crystallization of a molten sphere, J. Crystal Growth, 74 (1986), pp. 642-655.

[40] L.M. Hocking AND A.D. Rivers, The spreading of a drop by capillary action, J. Fluid Mech., 121 (1982), pp. 425-442.

[41] R.L. Hoffman, A study of the advancing interface I. Interface shape in liquid-gas systems, J. Coll. Int. Sci., 50 (1975), pp. 228-241.

[42] G. Mchale, S.M. Rowan, And M.I. Newton, Frenkel's method and the spreading of small spherical droplets, J. Phys. D.: Appl. Phys., 27 (1994), pp. 2619-2623.

[43] A.L. Bertozzi, A. Münch, X. Fanton, and A.M. Cazabat, Contact line stability and "undercompressive shocks" in driven thin film flow, Phys. Rev. Lett., 81 (1998), pp. 5169-5172.

[44] G.I. Barenblatt, E. Beretta, and M. Bertsch, The problem of the spreading of a liquid film along a solid surface: A new mathematical formulation, Proc. Natl. Acad. Sci. USA, 94 (1997), pp. 10024-10030.

[45] J.V. Wehausen, The motion of floating bodies, Annu. Rev. Fluid Mech., 3 (1971), pp. 237-268.

[46] H.S. Carslaw and J.C. Jaeger, Conduction of Heat in Solids, Oxford University Press, New York, 1959.

[47] K.E. Brenan, S.L. Campbell, and L.R. Petzold, Numerical Solution of Initial-Value Problems in Differential-Algebraic Equations, Classics Appl. Math. 14, SIAM, Philadelphia, PA, 1995 . 